\title{
A list of oribatid mites (Acari, Oribatida) of Vietnam
}

\author{
Sergey G. Ermilov' \\ I Tyumen State University, Tyumen, Russia \\ Corresponding author: Sergey G. Ermilov (ermilovacari@yandex.ru)
}

Academic editor: VladimirPesic | Received 14October 2014| Accepted 15 November 2015 | Published 16 December 2015

http://zoobank.org/4D74756E-9D67-45AD-98B3-F13029BC2020

Citation: Ermilov SG (2015) A list of oribatid mites (Acari, Oribatida) of Vietnam. ZooKeys 546: 61-85. doi: 10.3897/ zookeys. 546.6188

\begin{abstract}
A species list of identified oribatid mite taxa (Acari, Oribatida) in the fauna of Vietnam is provided. During 1967-2015, a total of 535 species/subspecies from 222 genera and 81 families was registered. Of these, 194 species/subspecies were described as new for science from Vietnam.
\end{abstract}

\section{Keywords}

Oribatid mites, species list, fauna, Vietnam

\section{Introduction}

The first data on oribatid mite fauna (Acari, Oribatida) of Vietnam were presented by Balogh and Mahunka (1967). Subsequent studies of oribatids during the next 40 years were fragmentary in character, and the main papers on descriptions of new taxa and new records are the following: Rajski and Szudrowicz (1974), Golosova $(1983,1984)$, Vu et al. (1985, 1987), Jeleva and Vu (1987), Mahunka (1987, 1988, 1989), Niedbała (1989, 2000, 2004), Vu (1990, 1993, 1994), Starý (1993), Vu and Thi (1995), Krivolutsky (1998), Vu and Nguyen (2000, 2005), and Pavlichenko (2001). 
Until 2007, only 195 species were recorded in Vietnam. Vu (2007) summarized this data, but his paper omitted more than 50 species, and did not consider synonyms and modern systematics.

A significant increase on oribatid taxonomic knowledge for Vietnam was contributed by the author and colleagues, which is based on material collected during joint Russian-Vietnamese expeditions in 2006-2014: Ermilov and Anichkin (2010, 2011ak, 2012a-d, 2013a-g, 2014a-g), Ermilov (2011, 2013), Ermilov et al. (2011a-c, 2012af, 2013a-c, 2014a-f), Niedbała and Ermilov (2013, 2014), Anichkin et al. (2014). At present, Vietnamese oribatid mites have been actively studied by several researchers (Ermilov 2015a, b; Ermilov and Anichkin 2015a, b; Ermilov and Bayartogtokh 2015; Ermilov and Corpuz-Raros 2015; Fernandez et al. 2015a, b; Vu et al. 2015).

The objective of this paper is to list all identified oribatid mite taxa known from Vietnam to date (1 September 2015), to present primary references (in square brackets) on descriptions of new species or new findings for each species, and to provide the subsequent faunistic and taxonomic studies.

General taxonomic system of Oribatida used in this paper mostly follows that of Subías (2004, online version 2015), Weigmann (2006), Norton and Behan-Pelletier (2009), and Schatz et al. (2011).

\section{Species list}

\section{Acaronychidae}

Loftacarus siefi Lee, 1981 [Vu et al. 2014]

Stomacarus ciliosus Luxton, 1982 [Vu et al. 2014]

S. leei Mahunka, 1989 [Vu et al. 2014]

\section{Ctenacaridae}

Ctenacarus araneola (Grandjean, 1932) [Ermilov and Anichkin 2013e]

\section{Cosmochthoniidae}

Cosmochthonius (Cosmochthonius) lanatus (Michael, 1885) [Vu et al. 1985]

\section{Sphaerochthoniidae}

Sphaerochthonius splendidus (Berlese, 1904) [Ermilov et al. 2012c]

\section{Hypochthoniidae}

Eohypochthonius (Eohypochthonius) crassisetiger Aoki, 1959 [Ermilov et al. 2012c]

E. (Eohypochthonius) gracilis Jacot, 1936 [Nguyen and Vu 2012]

Malacoangelia remigera Berlese, 1913 [Ermilov et al. 2012b]

\section{Lohmanniidae}

Annectacarus unilateralis Hammer, 1973 [Ermilov and Anichkin 2015a]

Bedoslomannia anneae Fernandez, Theron, Rolland \& Castillo, 2015 [Fernandez et al. 2015a]

Haplacarus pairathi Aoki, 1965 [Nguyen and Vu 2012]

Javacarus (Javacarus) kuehnelti Balogh, 1961 [Vu et al. 1985]

Lohmannia (Lohmannia) javana Balogh, 1961 [Vu et al. 1985] 
L. (Carolohmannia) monosetosa Ermilov \& Anichkin, 2014 [Ermilov and Anichkin 2014d]

Meristacarus africanus annobonensis Pérez-Ínigo, 1969 [Ermilov and Anichkin 2015a]

M. madagascarensis obscurus Aoki, 1965 [Golosova 1983]

M. sundensis Hammer, 1979 [Ermilov and Anichkin 2011i]

Mixacarus (Mixacarus) exilis Aoki, 1970 [Ermilov and Anichkin 2011i]

M. (Mixacarus) foliifer Golosova, 1984 [Golosova 1984]

Paillacarus aciculatus (Berlese, 1908) [Vu et al. 1987]

P. benenensis Vu, Ermilov \& Dao, 2010 [Vu et al. 2010]

P. cornutus Sarkar \& Subías, 1984 [Ermilov and Anichkin 2011i]

P. gueyeae (Pérez-Ínigo, 1989) [Nguyen and Vu 2012]

P. hirsutus (Aoki, 1961) (=Papillacarus arboriseta Jeleva \& Vu, 1987 - Ermilov et al. 2011a) [Golosova 1983]

P. indistinctus Ermilov, Anichkin \& Wu, 2012 [Ermilov et al. 2012b]

P. luteus Ermilov, 2015 [Ermilov 2015b]

P. polygonatus Ermilov \& Anichkin, 2011 [Ermilov and Anichkin 2011i]

P. polysetosus Ermilov, Anichkin \& Wu, 2012 [Ermilov et al. 2012b]

P. ramosus Balogh, 1961 [Ermilov and Anichkin 2011i]

P. undirostratus Aoki, 1965 [Vu and Nguyen 2000]

P. whitteni Fernandez, Theron, Rolland \& Leiva, 2015 [Fernandez et al. 2015b]

Paulianacarus (Paulianacarus) vietnamese Fernandez, Theron, Rolland \& Castillo, 2015 [Fernandez et al. 2015a]

\section{Nehypochthoniidae}

Nehypochthonius porosus Norton \& Metz, 1980 [Ermilov and Anichkin 2013e]

\section{Epilohmanniidae}

Epilohmannia crassisetosa Ermilov \& Anichkin, 2012 [Ermilov and Anichkin 2012b]

E. cylindrica (Berlese, 1904) [Vu et al. 1985]

E. dimorpha Wallwork, 1962 [Nguyen and Vu 2012]

E. minuta pacifica Aoki, 1965 [Ermilov et al. 2012c]

E. ornata Mahunka, 1993 [Vu et al. 2014]

E. spathulata Aoki, 1970 [Golosova 1983]

Epilohmannoides xena (Mahunka, 1983) [Vu et al. 2012]

\section{Protoplophoridae}

Arthrhoplophora vulpes Berlese, 1916 [Ermilov et al. 2012c]

\section{Mesoplophoridae}

Apoplophora minuscula Niedbała, 2013 [Niedbała and Ermilov 2013]

A. pantotrema (Berlese, 1913) [Golosova 1983]

\section{Oribotritiidae}

Austrotritia lebronneci (Jacot, 1934) [Niedbała and Ermilov 2014]

A. saraburensis Aoki, 1965 [Niedbała 2004] 
Indotritia javensis Sellnick, 1923 [as Indotritia completa Mahunka, 1987 - Mahunka 1987]

Oribotritia bulbifer (Mahunka, 1987) [Ermilov et al. 2012c]

O. paraaokii Niedbała, 2000 [Niedbała 2000]

O. submolesta Niedbała, 2000 [Niedbała 2000]

\section{Euphthiracaridae}

Acrotritia aokii (Niedbała, 2000) [Niedbała 2000]

A. ardua (Koch, 1841) [Vu et al. 1985]

A. duplicata (Grandjean, 1953) [Vu et al. 1985]

A. hyeroglyphica (Berlese, 1916) [as Rhysotritia hauseri Mahunka, 1991 - Vu et al. 2012]

A. otaheitensis (Hammer, 1972) [Starý 1993]

A. paragranulata Niedbała, 2014 [Niedbała and Ermilov 2014]

A. proxima Niedbała, 2013 [Niedbała and Ermilov 2013]

A. sinensis Jacot, 1923 [as Rhysotritia rasile Mahunka, 1982 - Vu et al. 1987]

A. vestita (Berlese, 1913) [as Rhysotritia comteae Mahunka, 1983 - Niedbała 2000]

Euphthiracarus (Euphthiracarus) foveolatus Aoki, 1980 [Niedbała 2004]

E. (Euphthiracarus) labyrinthicus Starý, 1993 [Starý 1993]

E. (Euphthiracarus) quasitakahashii Niedbała, 2014 [Niedbała and Ermilov 2014]

E. (Euphthiracarus) parareticulatus Niedbała, 2000 [Niedbała 2000]

E. (Euphthiracarus) vietnamicus Starý, 1993 [Starý 1993]

E. (Parapocsia) medius Niedbała, 2014 [Niedbała and Ermilov 2014]

Mesotritia maerkeli Sheals, 1965 [Niedbała and Ermilov 2014]

M. spinosa Aoki, 1980 [Starý 1993]

Microtritia minima (Berlese, 1904) [Niedbała 2000]

M. tropica Märkel, 1964 [Starý 1993]

\section{Synichotritiidae}

Sabahtritia dongnaiensis Niedbała, 2013 [Niedbała and Ermilov 2013]

\section{Steganacaridae}

Arphthiracarus parasentus Niedbała, 2000 [Niedbała 2000]

A. tubulus (Hammer, 1972) [Ermilov et al. 2012c]

Atropacarus (Atropacarus) phyllophorus (Berlese, 1904) [Vu et al. 1985]

A. (Atropacarus) striculus (Koch, 1935) [Niedbała 2000]

A. (Hoplophorella) cucullatus (Ewing, 1909) [Ermilov et al. 2012c]

A. (Hoplophorella) hamatus (Ewing, 1909) [as Hoplophorella cuneiseta Mahunka, 1988 - Mahunka 1988; also as Atropacarus floridae Jacot, 1933 - Niedbała 2000]

A. (Hoplophorella) stilifer (Hammer, 1961) [Niedbała 2000]

A. (Hoplophorella) vitrinus (Berlese, 1913) [Golosova 1983]

Austrophthiracarus evexus (Niedbała, 2000) [Niedbała 2000]

Austrophthiracarus pullus (Niedbała, 1989) [Ermilov et al. 2012c]

A. sentus Niedbała, 1989 [Niedbała 1989]

Plonaphacarus kugohi (Aoki, 1959) [Niedbała 2000] 
P. insignitus Niedbała, 1989 [Niedbała 1989]

P. scrupeus Niedbała, 1989 [Niedbała 1989]

Protophthiracarus finitima Niedbała, 2002 [Vu 2013]

Steganacarus (Rhacaplacarus) spinus Niedbała, 2014 [Niedbała and Ermilov 2014]

\section{Phthiracaridae}

Hoplophthiracarus clavatus Niedbała, 2014 [Niedbała and Ermilov 2014]

H. pakistanensis Hammer, 1977 [Nguyen and Vu 2012]

H. stigmosus Niedbała, 2000 [Niedbała 2000]

Notophthiracarus nitidus (Pérez-Ínigo \& Baggio, 1988) [Nguyen and Vu 2012]

N. perparvus Niedbała, 1989 [Niedbała 1989]

Phthiracarus abstemius Niedbała, 1989 [Niedbała 1989]

P. crispus Hammer, 1972 [Niedbała 2000]

P. invenustus Niedbała, 2000 [Niedbała 2000]

P. paucus Niedbała, 1991 [Niedbała 2000]

P. pygmaeus Balogh, 1958 [Niedbała 2000]

\section{Trhypochthoniidae}

Afronothrus incisivus Wallwork, 1961 [Ermilov et al. 2012a]

Allonothrus russeolus Wallwork, 1960 [Nguyen and Vu 2012]

Archegozetes longisetosus Aoki, 1965 [Vu 1990]

\section{Malaconothridae}

Malaconothrus dorsofoveolatus Hammer, 1979 [Ermilov et al. 2012c]

Tyrphonothrus adilatatus (Ermilov, Anichkin \& Tolstikov, 2014) [Ermilov et al. 2014a]

T. angustirostrum (Hammer, 1966) [Nguyen and $\mathrm{Vu} 2012$ ]

T. geminus (Hammer, 1972) [Ermilov et al. 2012c]

T. variosetosus (Hammer, 1971) [Ermilov et al. 2012b]

\section{Nothridae}

Nothrus baviensis Krivolutsky, 1998 [Krivolutsky 1998]

N. gracilis Hammer, 1961 [Vu et al. 2012]

N. montanus Krivolutsky, 1998 [Krivolutsky 1998]

N. oceanicus Sellnick, 1959 [Vu et al. 2014]

N. shapensis Krivolutsky, 1998 [Krivolutsky 1998]

\section{Crotoniidae}

Heminothrus apophysiger Hammer, 1969 [Ermilov and Anichkin 2014d]

\section{Nanhermanniidae}

Cosmohermannia robusta (Aoki, 1994) [Ermilov and Anichkin 2011i]

Cyrthermannia vicinicornuta Aoki, 1965 [Ermilov and Anichkin 2011i]

Masthermannia mammillaris (Berlese, 1904) [Ermilov and Anichkin 2011i]

Nanhermannia thainensis Aoki, 1965 [Vu 1993]

\section{Hermanniidae}

Phyllhermannia bimaculata Hammer, 1979 [Ermilov and Anichkin 2014f]

P. forsteri Balogh, 1985 [Vu et al. 2014]

P. gladiata Aoki, 1965 [Vu and Nguyen 2005] 
P. javensis Hammer, 1979 [Nguyen and Vu 2012]

P. similis Balogh \& Mahunka, 1967 [Balogh and Mahunka 1967]

\section{Hermanniellidae}

Hermanniella bugiamapensis Ermilov \& Bayartogtokh, 2015 [Ermilov and Bayartogtokh 2015]

H. aristosa Aoki, 1965 [Ermilov and Anichkin 2014d]

H. orbiculata Hammer, 1979 [Vu et al. 2014]

H. thani Mahunka, 1987 [Mahunka 1987]

\section{Plasmobatidae}

Plasmobates asiaticus Aoki, 1973 [Ermilov and Anichkin 2014d]

\section{Neoliodidae}

Neoliodes theleproctus (Hermann, 1804) [Vu et al. 1985]

Platyliodes japonicus Aoki, 1979 [Nguyen and Vu 2012]

\section{Pheroliodidae}

Pheroliodes longiceps Balogh \& Mahunka, 1966 [Vu et al. 2014]

\section{Licnodamaeidae}

Hexachaetoniella dispersa (Balogh, 1985) [Nguyen and Vu 2012]

Pedrocortesella dongnaiensis Ermilov \& Anichkin, 2014 [Ermilov and Anichkin 2014f]

P. pulchra Hammer, 1961 [Nguyen and Vu 2012]

P. temperata Balogh, 1985 [Nguyen and Vu 2012]

P. vietnamica Ermilov \& Anichkin, 2014 [Ermilov and Anichkin 2014f]

\section{Gymnodamaeidae}

Arthrodamaeus vietnamicus Ermilov \& Anichkin, 2011 [Ermilov and Anichkin 2011b]

Gymnodamaeus adpressus (Aoki \& Fujikawa, 1971) [Vu et al. 2008]

\section{Damaeidae}

Belba corynopus (Hermann, 1804) [Vu 1993]

Metabelba orientalis Balogh \& Mahunka, 1967 [Balogh and Mahunka 1967]

Tectodamaeus heterotrichus Ermilov \& Anichkin, 2014 [Ermilov and Anichkin 2014d]

\section{Cepheidae}

Sphodrocepheus tuberculatus Mahunka, 1988 [Mahunka 1988]

\section{Astegistidae}

Cultroribula bicuspidata Mahunka, 1978 [Ermilov et al. 2012c]

C. lata Aoki, 1961 [Golosova 1983]

Furcoppia (Furcoppia) cattienica Ermilov \& Anichkin, 2012 [Ermilov and Anichkin 2012b]

F. (Furcoppia) parva Balogh \& Mahunka, 1967 [Balogh and Mahunka 1967]

\section{Peloppiidae}

Austroceratoppia japonica Aoki, 1984 [Ermilov and Anichkin 2014d]

Ceratoppia bipilis (Hermann, 1804) [Ermilov and Anichkin 2014d]

C. crassiseta Balogh \& Mahunka, 1967 [Balogh and Mahunka 1967] 


\section{Gustaviidae}

Gustavia longicornis (Berlese, 1904) [Ermilov and Anichkin 2014d]

\section{Zetorchestidae}

Zetorchestes equestris Berlese, 1908 [Golosova 1993]

Z. saltator Oudemans, 1915 [Vu 1990]

Z. transvaalensis Coetzee, 1989 [Nguyen and Vu 2012]

Z. phylliferus Mahunka, 1983 [Vu et al. 2014]

\section{Ctenobelbidae}

Ctenobelba (Berndamerus) bugiamapensis Ermilov, Shtanchaeva, Subías \& Anichkin, 2014 [Ermilov et al. 2014f]

\section{Amerobelbidae}

Roynortonia vietnamica Ermilov, 2011 [Ermilov 2011]

\section{Eremulidae}

Austroeremulus glabrus Mahunka, 1985 [Nguyen and Vu 2012]

Eremulus avenifer Berlese, 1913 [Balogh and Mahunka 1967]

E. flagellifer Berlese, 1908 [Nguyen and Vu 2012]

E. spinosus Ermilov \& Anichkin, 2011 [Ermilov and Anichkin 2011d]

E. truncatus Hammer, 1971 [Golosova 1983]

Mahunkana bifurcata (Mahunka, 1987) [Mahunka 1987]

M. japonica (Aoki \& Karasawa, 2007) [Ermilov et al. 2012c]

Reteremuloides bifurcatus Mahunka, 1989 [Ermilov and Bayartogtokh 2015]

\section{Damaeolidae}

Fosseremus laciniatus (Berlese, 1905) [Ermilov et al. 2012c]

Gressittolus marginatus Balogh, 1970 [Ermilov et al. 2012c]

\section{Hungarobelbidae}

Costeremus ornatus Aoki, 1970 [Vu et al. 2014]

\section{Eremobelbidae}

Eremobelba bella Hammer, 1982 [Nguyen and Vu 2012]

E. bellicosa Balogh \& Mahunka, 1967 [Balogh and Mahunka 1967]

E. breviseta Balogh, 1968 [Ermilov et al. 2012c]

E. capitata Berlese, 1913 [Vu et al. 1985]

E. hamata Hammer, 1961 [Nguyen and Vu 2012]

E. japonica Aoki, 1959 [Vu et al. 2011]

\section{Heterobelbidae}

Heterobelba stellifera stellifera Okayama, 1980 [Ermilov et al. 2012a]

H. stellifera formosana Aoki, 1990 [Vu et al. 2014]

\section{Basilobelbidae}

Basilobelba maidililae Fernandez, Theron, Rolland \& Leiva, 2015 [Fernandez et al. 2015b]

B. parmata Okayama, 1980 [Ermilov et al. 2012b]

$B$. retiaria (Warburton, 1912) [Ermilov and Anichkin 2013g]

Xyphobelba hamanni Csiszár, 1961 [Ermilov and Anichkin 2014d] 


\section{Platyameridae}

Gymnodampia crassisetiger (Aoki, 1984) [Ermilov and Anichkin 2014d]

\section{Caleremaeidae}

Epieremulus bidupensis Ermilov \& Anichkin, 2014 [Ermilov and Anichkin 2014d]

\section{Eremellidae}

Eremella vestita Berlese, 1913 [Vu and Nguyen 2000]

\section{Arceremaeidae}

Tecteremaeus incompletus Mahunka, 1988 [Ermilov and Bayartogtokh 2015]

T. hauseri Mahunka, 1982 [Ermilov et al. 2012c]

\section{Oppiidae}

Acroppia processigera (Balogh \& Mahunka, 1967) [Nguyen and Vu 2012]

Arcoppia arcualis (Berlese, 1913) [Balogh and Mahunka 1967]

A. corniculifera (Mahunka, 1978) [Nguyen and Vu 2012]

A. hammerae Rodríguez \& Subías, 1984 [Vu and Nguyen 2005]

A. incerta Balogh \& Balogh, 1983 [Vu et al. 2014]

A. longisetosa Balogh, 1962 [Vu 1990]

A. robustia (Berlese, 1913) [Balogh and Mahunka 1967]

A. serrutala (Balogh \& Mahunka, 1980) [Ermilov and Bayartogtokh 2015]

A. viperea (Aoki, 1959) [as Arcoppia baloghi Rodríguez \& Subías, 1984 - Vu and Nguyen 2005]

A. waterhousei Balogh \& Balogh, 1983 [Nguyen and Vu 2012]

Belloppia shealsi Hammer, 1968 [Vu et al. 2012]

Berniniella (Berniniella) bicarinata (Paoli, 1908) [Vu et al. 1985]

Brachioppiella (Brachioppiella) biseriata (Balogh \& Mahunka, 1975) [Vu et al. 2012]

Congoppia deboissezoni (Balogh \& Mahunka, 1966) [Vu et al. 2012]

Cryptoppia elongata Csiszár, 1961 [Vu et al. 1985]

Cycloppia restata (Aoki, 1963) [Ermilov and Anichkin 2013f]

Discoppia (Cylindroppia) cylindrica (Pérez-Í́nigo, 1965) [Ermilov et al. 2012b]

Elaphoppia quadripilosa (Balogh, 1961) [Ermilov and Anichkin 2014d]

Helioppia sol (Balogh, 1959) [Vu et al. 2012]

Karenella (Karenella) acuta (Csiszár, 1961) [Vu et al. 1985]

Kokoppia dendricola (Jeleva \& Vu, 1987) [Jeleva and Vu 1987]

Lanceoppia (Lancelalmoppia) becki Hammer, 1968 [Nguyen and Vu 2012]

L. (Lanceoppia) translucens (Mahunka, 1985) [Vu et al. 2014]

Lasiobelba (Lasiobelba) kuehnelti (Csiszár, 1961) [Vu et al. 1985]

L. (Lasiobelba) remota Aoki, 1959 [Balogh and Mahunka 1967]

L. (Lasiobelba) vietnamica (Balogh, 1983) [as Oppia remota Aoki, 1959 - Balogh and Mahunka 1967]

Lineoppia microseta Ermilov \& Anichkin, 2011 [Ermilov and Anichkin 2011g] Lyroppia dongnaiensis Ermilov \& Anichkin, 2013 [Ermilov and Anichkin 2013f] Microppia minus (Paoli, 1908) [Ermilov et al. 2012b]

Multioppia (Multioppia) pseudoglabra Ermilov, 2015 [Ermilov 2015b] 
M. (Multioppia) tamdao Mahunka, 1988 [Mahunka 1988]

Multipulchroppia pectinata (Balogh \& Mahunka, 1967) [Balogh and Mahunka 1967]

M. similis (Hammer, 1979) [Nguyen and Vu 2012]

Neoamerioppia (Neoamerioppia) vietnamica (Mahunka, 1988) [Mahunka 1988]

Oppiella (Oppiella) nova (Oudemans, 1902) [Golosova 1983]

Oxybrachioppia barbata (Choi, 1986) [Ermilov et al. 2012c]

Oxyoppia (Aciculoppa) clavata (Aoki, 1983) [Vu et al. 2008]

Pulchroppia elegans Hammer, 1979 [Ermilov et al. 2012c]

P. granulata Mahunka, 1988 [Mahunka 1988]

P. roynortoni Ermilov \& Anichkin, 2011 [Ermilov and Anichkin 2011g]

Ramusella (Ramusella) chulumaniensis (Hammer, 1958) [Ermilov et al. 2012b]

R. (Ramusella) clavipectinata (Michael, 1885) [Vu et al. 1985]

R. (Ramusella) pocsi Balogh \& Mahunka, 1967 [Balogh and Mahunka 1967]

R. (Insculptoppia) elliptica (Berlese, 1908) [Ermilov et al. 2012c]

R. (Insculptoppia) insculpta (Paoli, 1908) [Vu 1993]

Ramuselloppia vietnamica Ermilov \& Anichkin, 2013 [Ermilov and Anichkin 2013b]

Striatoppia lanceolata Hammer, 1972 [Ermilov et al. 2013a]

S. madagascarensis Balogh, 1961 [Vu et al. 2012]

S. opuntiseta Balogh \& Mahunka, 1968 [Mahunka 1988]

S. papillata Balogh \& Mahunka, 1966 [Vu et al. 1985]

Taiwanoppia (Taiwanoppia) hungarorum (Mahunka, 1988) [Mahunka 1988]

\section{Granuloppiidae}

Gigantoppia zryanini Ermilov \& Anichkin, 2011 [Ermilov and Anichkin 2011e] Granuloppia kamerunensis Mahunka, 1974 [Nguyen and Vu 2012]

G. vietnamensis Ermilov \& Bayartogtokh, 2015 [Ermilov and Bayartogtokh 2015] Hammerella (Parawoasella) bayartogtokhi Ermilov, Shtanchaeva, Subías \& Anichkin, 2012 [Ermilov et al. 2012f]

\section{Machuellidae}

Machuella lineata Hammer, 1973 [Ermilov et al. 2012b]

\section{Suctobelbidae}

Allosuctobelba vietnamensis Ermilov \& Anichkin, 2014 [Ermilov and Anichkin 2014d]

Novosuctobelba (Novosuctobelba) vietnamica Balogh \& Mahunka, 1967 [Balogh and Mahunka 1967]

Suctobelbata bituberculata Ermilov \& Anichkin, 2013 [Ermilov and Anichkin 2013c]

Suctobelbella (Suctobelbella) elegantissima (Hammer, 1979) [Golosova 1983]

S. (Suctobelbella) finlayi (Balogh \& Mahunka, 1980) [Nguyen and Vu 2012]

S. (Suctobelbella) latirostris (Strenzke, 1950) [Vu 1990]

S. (Suctobelbella) longicuspis Jacot, 1937 [Nguyen and Vu 2012]

S. (Flagrosuctobelba) elegantula (Hammer, 1958) [Ermilov et al. 2012c] 
S. (Flagrosuctobelba) parallelodentata Hammer, 1979 [Ermilov et al. 2012c]

S. (Flagrosuctobelba) semiplumosa (Balogh \& Mahunka, 1967) [Balogh and Mahunka 1967]

S. (Flagrosuctobelba) subtrigona (Oudemans, 1900) [Vu et al. 2012]

S. (Ussuribata) bivittata (Hammer, 1979) [Ermilov and Anichkin 2014d]

S. (Ussuribata) multituberculata Balogh \& Mahunka, 1967 [Balogh and Mahunka 1967]

S. (Ussuribata) phylliformis Ermilov, Shtanchaeva \& Subías, 2014 [Ermilov et al. 2014d]

S. (Ussuribata) sexsetosa (Hammer, 1979) [Ermilov and Anichkin 2013d]

S. (Ussuribata) variosetosa (Hammer, 1961) [Golosova 1983]

Suctobelbila minima Hammer, 1979 [Golosova 1983]

S. multituberculata Hammer, 1979 [Ermilov et al. 2012c]

S. scutata Hammer, 1972 [Ermilov and Anichkin 2014d]

S. transrugosa (Mahunka, 1986) [Nguyen and Vu 2012]

\section{Oxyameridae}

Oxyamerus aokii Balogh, 1968 [Ermilov and Anichkin 2014d]

O. hyalinus Hammer, 1979 [Ermilov and Anichkin 2014d]

O. truncatus Hammer, 1979 [Ermilov and Anichkin 2014d]

\section{Dampfiellidae}

Dampfiella angusta Hammer, 1979 [Ermilov and Anichkin 2014d]

\section{Otocepheidae}

Basiceramerus igorotus Corpuz-Raros and Gruèzo, 2011 [Ermilov and Anichkin $2013 \mathrm{~g}]$

Eurostocepheus aquilinus Aoki, 1965 [Ermilov et al. 2012c]

Dolicheremaeus aokii Balogh \& Mahunka, 1967 [Balogh and Mahunka 1967]

D. baloghi Aoki, 1967 [Ermilov and Bayartogtokh 2005]

D. bartkei Rajski \& Szudrowicz, 1974 [Rajski and Szudrowicz 1974]

D. bruneiensis Aoki, 1967 [Ermilov and Bayartogtokh 2005]

D. bugiamapensis Ermilov, Anichkin \& Wu, 2012 [Ermilov et al. 2012a]

D. capillatus (Balogh, 1959) [Nguyen and Vu 2012]

D. contactus Ermilov \& Anichkin, 2013 [Ermilov and Anichkin 2013d]

D. damaeoides (Berlese, 1913) [Ermilov and Anichkin 2014d]

D. donacunarensis Ermilov \& Anichkin, 2014 [Ermilov and Anichkin 2014g]

D. dwalteri Ermilov \& Anichkin, 2014 [Ermilov and Anichkin 2014g]

D. insolitus Ermilov \& Anichkin, 2014 [Ermilov and Anichkin 2014d]

D. junichiaokii Subías, 2010 (=Dolicheremaeus magnus Aoki, 2006) [Ermilov and Anichkin 2013g]

D. inaequalis Balogh \& Mahunka, 1967 [Balogh and Mahunka 1967]

D. lineolatus Balogh \& Mahunka, 1967 [Balogh and Mahunka 1967]

D. orientalis (Aoki, 1965) [Vu 1994]

D. ornatus Balogh \& Mahunka, 1967 [Balogh and Mahunka 1967]

D. philippinensis Aoki, 1967 [Vu et al. 2014] 
D. sabahnus Mahunka, 1988 [Nguyen and Vu 2012]

Fissicepheus (Fissicepheus) elegans Balogh \& Mahunka, 1967 [Balogh and Mahunka 1967]

F. (Fissicepheus) striganovae Ermilov \& Anichkin, 2014 [Ermilov and Anichkin 2014d]

Leptotocepheus murphyi (Mahunka, 1989) [Ermilov and Bayartogtokh 2015]

Megalotocepheus (Archegotocepheus) crinitus Berlese, 1905 [Ermilov and Anichkin 2014d]

M. (Archegotocepheus) singularis (Mahunka, 1988) [Nguyen and Vu 2012]

Otocepheus (Otocepheus) spatulatus Mahunka, 2000 [Ermilov and Anichkin 2013c]

O. (Acrotocepheus) duplicornutus duplicornutus Aoki, 1965 [Vu and Nguyen 2005]

O. (Acrotocepheus) duplicornutus discrepans Balogh \& Mahunka, 1967 [Balogh and Mahunka 1967]

O. (Acrotocepheus) excelsus Aoki, 1965 [Ermilov and Anichkin 2013d]

O. (Acrotocepheus) triplicicornutus Balogh \& Mahunka, 1967 [Balogh and Mahunka 1967]

O. (Acrotocepheus) vietnamicus Ermilov \& Anichkin, 2011 [Ermilov and Anichkin 2011e]

Papillocepheus primus Ermilov, Anichkin \& Tolstikov, 2014 [Ermilov et al. 2014b] Pseudotocepheus setiger (Hammer, 1972) [Ermilov et al. 2013a]

Umashtanchaeviella plethotricha Ermilov, Anichkin \& Tolstikov, 2014 [Ermilov et al. 2014c]

\section{Carabodidae}

Aokiella florens Balogh \& Mahunka, 1967 [Balogh and Mahunka 1967]

A. rotunda Hammer, 1979 [Ermilov et al. 2012b]

A. xuansoni Vu, Ermilov \& Dao, 2010 [Vu et al. 2010]

Austrocarabodes (Austrocarabodes) alveolatus Hammer, 1973 [Vu et al. 2014]

A. (Austrocarabodes) falcatus Hammer, 1973 [Nguyen and Vu 2012]

A. (Austrocarabodes) szentivanyi (Balogh \& Mahunka, 1967) [Balogh and Mahunka 1967]

A. (Austrocarabodes) vaucheri Mahunka, 1984 [Nguyen and Vu 2012]

A. (Uluguroides) polytrichus Balogh \& Mahunka, 1978 [Vu et al. 2015]

Carabodes (Klapperiches) mikhaetandreorum Ermilov \& Anichkin, 2013 [Ermilov and Anichkin 2013g]

C. (Klapperiches) samoensis Balogh \& Balogh, 1986 [Ermilov and Anichkin 2013d]

C. (Klapperiches) strinovichi Balogh \& Mahunka, 1978 [Vu et al. 2014]

C. (Phyllocarabodes) inopinatus (Mahunka, 1985) [Nguyen and Vu 2012]

C. (Phyllocarabodes) schatzi Subías, 2010 [as Phyllocarabodes ornatus Balogh, 1986 - Nguyen and Vu 2012]

Chistyakovella insolita Ermilov, Aoki \& Anichkin, 2013 [Ermilov et al. 2013c]

Gibbicepheus (Gibbicepheus) baccanensis Jeleva \& Vu, 1987 [Jeleva and Vu 1987]

G. (Gibbicepheus) fenestralis Hammer, 1979 [Golosova 1983]

G. (Gibbicepheus) latohumeralis Hammer, 1982 [Ermilov and Anichkin 2015a] 
Yoshiobodes (Yoshiobodes) irmayi (Balogh \& Mahunka, 1969) [as Yoshiobodes aokii Mahunka, 1987 - Ermilov and Anichkin 2013c]

Y. (Yoshiobodes) neotrichorostralis Ermilov, Shtanchaeva, Subías \& Anichkin, 2014 [Ermilov et al. 2014e]

Y. (Dongnaibodes) biconcavus Ermilov, Shtanchaeva, Subías \& Anichkin, 2014 [Ermilov et al. 2014e]

Y. (Dongnaibodes) hexasetosus Ermilov, Shtanchaeva, Subías \& Anichkin, 2014 [Ermilov et al. 2014e]

\section{Nippobodidae}

Nippobodes monstruosus (Jeleva \& Vu, 1987) [Jeleva and Vu 1987]

\section{Tectocepheidae}

Tectocepheus elegans Ohkubo, 1981 [Vu et al. 2014]

T. minor Berlese, 1903 [as Tectocepheus cuspidentatus Knülle, 1954 - Vu 1993]

T. velatus (Michael, 1880) [Golosova 1983]

Tegeozetes tunicatus tunicatus Berlese, 1913 [Ermilov and Anichkin 2013f]

T. tunicatus breviclava Aoki, 1970 [Vu 2013]

\section{Tegeocranellidae}

Tegeocranellus martinezi Ermilov \& Anichkin, 2014 [Ermilov and Anichkin 2014d]

\section{Microtegeidae}

Microtegeus borhidii Balogh \& Mahunka, 1974 [Ermilov et al. 2012a]

M. cardosensis Pérez-Ínigo, 1985 [Vu et al. 2014]

M. cornutus Balogh, 1970 [Nguyen and $\mathrm{Vu}$ 2012]

M. quadristriatus Mahunka, 1984 [Nguyen and Vu 2012]

M. reticulatus Aoki, 1965 [Vu 1990]

\section{Cymbaeremaeidae}

Scapheremaeus ascissuratus Ermilov \& Anichkin, 2015 [Ermilov and Anichkin 2015b]

S. cellulatifer Mahunka, 1987 [Mahunka 1987]

S. crassus Mahunka, 1988 [Mahunka 1988]

S. fisheri Aoki, 1966 [Ermilov et al. 2012c]

S. foveolatus Mahunka, 1987 [Mahunka 1987]

\section{Licneremaeidae}

Licneremaeus licnophorus (Michael, 1882) [Vu et al. 2014]

L. polygonalis Hammer, 1971 [Ermilov et al. 2013a]

\section{Phenopelopidae}

Eupelops forsslundi (Balogh, 1959) [Vu et al. 1987]

Nesopelops intermedius Hammer, 1979 [Ermilov and Anichkin 2013g]

\section{Eremaeozetidae}

Mahunkaia bituberculata (Mahunka, 1983) [Nguyen and Vu 2012]

\section{Idiozetidae}

Idiozetes javensis Hammer, 1979 [Ermilov et al. 2012a]

\section{Limnozetidae}

Limnozetes pustulatus (Mahunka, 1987) [Mahunka 1987] 


\section{Microzetidae}

Berlesezetes ornatissimus (Berlese, 1913) [as Berlesezetes auxiliaris Grandjean, 1936 - Mahunka 1988]

Caucasiozetes frankeae Ermilov \& Anichkin, 2011 [Ermilov and Anichkin 2011e] Kaszabodes velatus Mahunka, 1988 [Mahunka 1988]

Schalleriella vietnamica Ermilov \& Anichkin, 2011 [Ermilov and Anichkin 2011a]

\section{Achipteriidae}

Achipteria (Achipteria) curta Aoki, 1970 [Vu 1993]

Anachipteria (Anachipteria) svetlanae Ermilov \& Anichkin, 2014 [Ermilov and Anichkin 2014d]

Austrachipteria phongnhae Ermilov \& Vu, 2012 [Ermilov and Vu 2012]

Campachipteria distincta (Aoki, 1959) [Vu and Nguyen 2000]

C. uenoi Aoki, 1995 [Ermilov and Anichkin 2014d]

Plakoribates asiaticus Ermilov \& Anichkin, 2013 [Ermilov and Anichkin 2013a]

\section{Tegoribatidae}

Ceratobates cangioensis Ermilov \& Anichkin, 2015 [Ermilov and Anichkin 2015a] Tegoribates americanus Hammer, 1958 [Vu et al. 2014]

\section{Oribatellidae}

Novoribatella minutisetarum Engelbrecht, 1986 [Nguyen and Vu 2012]

Ophidiotrichus ussuricus Krivolutsky, 1971 [Vu et al. 2014]

Oribatella (Oribatella) gerdweigmanni Ermilov \& Anichkin, 2012 [Ermilov and Anichkin 2012d]

O. (Oribatella) illuminata Hammer, 1961 [Vu et al. 2014]

O. (Oribatella) prolongata Hammer, 1961 [Vu et al. 2014]

O. (Oribatella) sculpturata Mahunka, 1987 [Mahunka 1987]

O. (Oribatella) umaetluisorum Ermilov \& Anichkin, 2012 [Ermilov and Anichkin 2012a]

\section{Heterozetidae}

Farchacarus philippinensis (Corpuz-Raros, 1979) [Golosova 1983]

\section{Ceratozetidae}

Ceratozetes (Ceratozetes) bicornis Hammer, 1967 [Golosova 1983]

C. (Ceratozetes) gracilis (Michael, 1884) [Golosova 1983]

C. (Ceratozetes) mediocris Berlese, 1908 [Golosova 1983]

Fuscozetes fuscipes (Koch, 1844) [Vu 1990]

Lepidozetes trifolius (Fujikawa, 1972) [Vu et al. 2014]

Sphaerozetes bugiamapensis Ermilov, Anichkin \& Wu, 2013 [Ermilov et al. 2013b]

\section{Punctoribatidae}

Allozetes africanus Balogh, 1958 [Golosova 1983]

A. pusillus (Berlese, 1913) [Mahunka 1988]

Lamellobates molecula (Berlese, 1916) [Golosova 1983; also as Lamellobates palustris Hammer, 1958 - Vu et al. 1985; as Lamellobates hauseri Mahunka, 1977 - Mahunka 1988]

L. ocularis Jeleva \& Vu, 1987 [Jeleva and Vu 1987] 
Paralamellobates misella (Berlese, 1910) [as Paralamellobates schoutedani (Balogh, 1959) - Vu et al. 1985; also as Paralamellobates ceylanicus (Oudemans, 1916) - Mahunka 1988]

Punctoribates hexagonus Berlese, 1908 [Vu 1990]

P. punctum (Koch, 1839) [Pavlichenko 1991]

\section{Chamobatidae}

Chamobates (Chamobates) javensis (Hammer, 1979) [Golosova 1983]

\section{Mochlozetidae}

Mochlozetes ryukyuensis Aoki, 2006 [Ermilov and Anichkin 2013d]

Unguizetes asiaticus Ermilov \& Anichkin, 2012 [Ermilov and Anichkin 2012b]

U. cattienensis Ermilov \& Anichkin, 2011 [Ermilov and Anichkin 2011e]

U. clavatus Aoki, 1967 [Golosova 1983]

U. latus Ermilov \& Anichkin, 2013 [Ermilov and Anichkin 2013d]

U. sphaerula (Berlese, 1905) [Ermilov et al. 2012c]

Uracrobates (Uracrobates) magniporosus Balogh \& Mahunka, 1967 [Balogh and Mahunka 1967]

\section{Oribatulidae}

Paraphauloppia gracilis (Hammer, 1958) [Nguyen and Vu 2012]

Zygoribatula pennata Grobler, 1993 [Nguyen and Vu 2012]

Z. prima Ermilov \& Anichkin, 2011 [Ermilov and Anichkin 2011k]

Z. undulata Berlese, 1916 [as Zygoribatula longiporosa Hammer, 1953 - Vu et al. 2012]

\section{Sellnickiidae}

Sellnickia caudata (Michael, 1908) [Ermilov et al. 2012c]

\section{Caloppiidae}

Zetorchella latior (Berlese, 1913) [Ermilov et al. 2012c]

Z. reticulata (Willmann, 1933) [Ermilov and Anichkin 2013g]

\section{Scheloribatidae}

Areozetes incertus Balogh, 1970 [Vu et al. 2014]

Cordiozetes olahi (Mahunka, 1987) [Mahunka 1987]

Euscheloribates (Euscheloribates) samsinaki Kunst, 1958 [Vu 1990]

E. (Trischeloribates) clavatus (Mahunka, 1988) [Mahunka 1988]

Exoribatula (Multoribates) longior (Hammer, 1958) [Ermilov and Anichkin 2014d]

Fijibates aelleni (Mahunka, 1988) [Ermilov et al. 2012c]

F. rostratus Hammer, 1971 [Ermilov et al. 2013a]

Liebstadia (Liebstadia) humerata Sellnick, 1928 [Vu 1993]

Perscheloribates (Perscheloribates) lanceolatus (Aoki, 1984) [Vu et al. 2008]

P. (Perscheloribates) luminosus (Hammer, 1961) [Ermilov and Anichkin 2014d]

P. (Perscheloribates) luteus (Hammer, 1962) [Nguyen and Vu 2012]

P. (Perscheloribates) minutus (Pletzen, 1965) [Golosova 1983]

Rhabdoribates siamensis Aoki, 1967 [Vu 1990]

Scheloribates (Scheloribates) crucisetus Jeleva \& Vu, 1987 [Jeleva and Vu 1987]

S. (Scheloribates) fimbriatus Thor, 1930 [Vu et al. 1985] 
S. (Scheloribates) flagellisetosus Ermilov \& Anichkin, 2014 [Ermilov and Anichkin 2014c]

S. (Scheloribates) kraepelini (Berlese, 1908) [Ermilov and Anichkin 2013e]

S. (Scheloribates) laevigatus (Koch, 1835) [Vu et al. 1985]

S. (Scheloribates) latipes (Koch, 1844) [Golosova 1983]

S. (Scheloribates) pallidulus (Koch, 1844) [Vu et al. 1985]

S. (Scheloribates) parvus Pletzen, 1963 [Vu et al. 2015]

S. (Scheloribates) praeincisus praeincisus (Berlese, 1910) [Golosova 1983]

S. (Scheloribates) praeincisus interruptus (Berlese, 1916) [Ermilov and Anichkin 2013g]

S. (Scheloribates) vulgaris Hammer, 1961 [Nguyen and Vu 2012]

S. (Bischeloribates) mahunkai Subías, 2010 [as Philoribates heterodactylus (Mahunka, 1988) - Nguyen and Vu 2012]

Tuberemaeus lineatus Balogh, 1970 [Nguyen and Vu 2012]

T. perforatoides Hammer, 1979 [Ermilov and Anichkin 2013d]

T. sculpturatus Mahunka, 1987 [Mahunka 1987]

T. singularis Sellnick, 1930 [Ermilov and Anichkin 2013g]

Vesiculobates silvaticus Hammer, 1979 [Ermilov and Anichkin 2014f]

\section{Oripodidae}

Brachyoripoda foveolata Balogh, 1970 [Ermilov et al. 2012c]

Cosmopirnodus tridactylus Mahunka, 1988 [Mahunka 1988]

Oripoda excavata Mahunka, 1988 [Mahunka 1988]

O. pinicola Aoki \& Ohkubo, 1974 [Vu et al. 2008]

Subpirnodus mirabilis Mahunka, 1988 [Mahunka 1988]

Truncopes moderatus variabilis Aoki \& Yamamoto, 2007 [Ermilov and Anichkin 2013f]

T. orientalis Mahunka, 1987 [Mahunka 1987]

\section{Haplozetidae}

Acutozetes rostratus Balogh, 1970 [Vu et al. 2014]

Indoribates (Indoribates) bicarinatus Ermilov \& Anichkin, 2014 [Ermilov and Anichkin 2014d]

I. (Indoribates) microsetosus Ermilov \& Anichkin, 2011 [Ermilov and Anichkin 2011j]

I. (Indoribates) nobilis (Golosova, 1984) [Golosova 1984]

I. (Indoribates) punctulatus (Sellnick, 1925) [as Indoribates panabokkei (Balogh, 1970) - Ermilov and Anichkin 2013e]

Haplozetes vindobonensis (Willmann, 1935) [Ermilov et al. 2012c]

Lauritzenia (Incabates) major (Aoki, 1967) [Vu et al. 2014]

L. (Magnobates) glagellifer (Hammer, 1967) [Vu 1990]

Peloribates (Peloribates) gressitti Balogh \& Mahunka, 1967 [Balogh and Mahunka 1967]

P. (Peloribates) guttatoides Hammer, 1979 [Ermilov and Anichkin 2014f]

P. (Peloribates) guttatus Hammer, 1979 [Vu et al. 2014] 
P. (Peloribates) kaszabi Mahunka, 1988 [Mahunka 1988]

P. (Peloribates) paraguayensis Balogh \& Mahunka, 1981 [Nguyen and Vu 2012]

P. (Peloribates) pseudoporosus Balogh \& Mahunka, 1967 [Balogh and Mahunka 1967]

P. (Peloribates) rangiroaensis Hammer, 1972 [Ermilov and Anichkin 2011k]

P. (Peloribates) ratubakensis Hammer, 1979 [Vu et al. 2014]

P. (Peloribates) spiniformis Ermilov \& Anichkin, 2011 [Ermilov and Anichkin 2011k]

P. (Peloribates) stellatus Balogh \& Mahunka, 1967 [Balogh and Mahunka 1967]

P. (Peloribates) tatyanae Ermilov \& Anichkin, 2014 [Ermilov and Anichkin 2014d]

Perxylobates brevisetosus Mahunka, 1988 [Mahunka 1988]

P. crassisetosus Ermilov \& Anichkin, 2011 [Ermilov and Anichkin 2011j]

P. guehoi Mahunka, 1978 [Nguyen and Vu 2012]

P. thanhoaensis Ermilov, Vu, Trinh \& Dao, 2011 [Ermilov et al. 2011c]

P. vermisetus (Balogh \& Mahunka, 1968) [Vu et al. 1985]

P. vietnamensis (Jeleva \& Vu, 1987) [Jeleva and Vu 1987]

Protoribates (Protoribates) capucinus Berlese, 1908 [Golosova 1983]

P. (Protoribates) cattienensis Ermilov \& Anichkin, 2011 [Ermilov and Anichkin $2011 \mathrm{j}]$

P. (Protoribates) dentatus (Berlese, 1883) [as Protoribates monodactylus (Haller, 1884) - Vu et al. 1985]

P. (Protoribates) gracilis (Aoki, 1982) [Vu and Nguyen 2000]

P. (Protoribates) lophotrichus (Berlese, 1904) [Vu et al. 1985]

P. (Protoribates) paracapucinus (Mahunka, 1988) [Ermilov et al. 2012c]

P. (Triaunguis) acutus (Hammer, 1979) [Golosova 1983]

P. (Triaunguis) bisculpturatus (Mahunka, 1988) [Vu et al. 2014]

P. (Triaunguis) duoseta (Hammer, 1979) [Nguyen and Vu 2012]

P. (Triaunguis) heterodactylus Ermilov \& Anichkin, 2011 [Ermilov and Anichkin 2011c]

P. (Triaunguis) maximus (Mahunka, 1988) [Mahunka 1988]

Setoxylobates (Setoxylobates) foveolatus Balogh \& Mahunka, 1967 [Balogh and Mahunka 1967]

S. (Polyxylobates) diversiporosus (Hammer, 1973) [Ermilov and Anichkin 2015a]

Trachyoribates irregularis (Balogh \& Mahunka, 1969) [Vu et al. 1985]

T. ovulum Berlese, 1908 [as Rostrozetes foveolatus Sellnick, 1925 - Golosova 1983; also as Rostrozetes areolatus (Balogh, 1958) - Vu et al. 1985; as Rostrozetes punctulifer Balogh \& Mahunka, 1979 - Vu et al. 1987; as Rostrozetes trimorphus Balogh \& Mahunka, 1979 - Vu 1990]

Transoribates agricola (Nakamura \& Aoki, 1989) [Ermilov and Anichkin 2014f]

Vilhenabates sinatus (Aoki, 1965) [Ermilov et al. 2012a]

\section{Parakalummidae}

Neoribates (Neoribates) aurantiacus (Oudemans, 1914) [Vu 1990]

N. (Neoribates) jacoti (Balogh \& Mahunka, 1967) [Balogh and Mahunka 1967] 
N. (Neoribates) monodactylus Ermilov \& Anichkin, 2014 [Ermilov and Anichkin 2014d]

N. (Neoribates) paratuberculatus Ermilov, Shtanchaeva \& Subías, 2014 [Ermilov et al. 2014d]

N. (Neoribates) spindleformis Ermilov \& Anichkin, 2012 [Ermilov and Anichkin 2012c]

\section{Galumnidae}

Allogalumna (Allogalumna) bipartita (Aoki \& Hu, 1993) [Ermilov and Anichkin 2013c]

A. (Allogalumna) costata Mahunka, 1996 [Vu et al. 2014]

A. (Allogalumna) monodactyla Ermilov \& Anichkin, 2014 [Ermilov and Anichkin 2014e]

A. (Allogalumna) multesima Grandjean, 1957 [Nguyen and Vu 2012]

A. (Allogalumna) paramachadoi Ermilov \& Anichkin, 2014 [Ermilov and Anichkin 2014d]

A. (Allogalumna) rotundiceps Aoki, 1996 [Ermilov and Anichkin 2014d]

A. (Allogalumna) upoluensis Hammer, 1973 [Vu et al. 2014]

A. (Globogalumna) biporosa Ermilov \& Anichkin, 2012 [Ermilov and Anichkin 2012c]

Dimidiogalumna azumai Aoki, 1996 [Nguyen et al. 2013]

D. grandjeani Ermilov \& Anichkin, 2014 [Ermilov and Anichkin 2014a]

Galumna (Galumna) aba Mahunka, 1989 [Mahunka 1989]

G. (Galumna) acutirostrum Ermilov \& Anichkin, 2010 [Ermilov and Anichkin 2010]

G. (Galumna) coronata Mahunka, 1992 [Vu et al. 2015]

G. (Galumna) discifera Balogh, 1960 [Nguyen and Vu 2012]

G. (Galumna) flabellifera Hammer, 1958 [as Galumna flabellifera orientalis Aoki, 1965 - Vu 1990]

G. (Galumna) kebangica Ermilov \& Vu, 2012 [Ermilov and Vu 2012]

G. (Galumna) khoii Mahunka, 1989 [Mahunka 1989]

G. (Galumna) lanceata (Oudemans, 1900) [Golosova 1983]

G. (Galumna) levisensilla Ermilov \& Anichkin, 2010 [Ermilov and Anichkin 2010]

G. (Galumna) microfissum Hammer, 1968 [Vu et al. 2014]

G. (Galumna) obvia (Berlese, 1914) [Vu 1993]

G. (Galumna) paracalcicola Ermilov \& Anichkin, 2014 [Ermilov and Anichkin 2014e]

G. (Galumna) parakazakhstani Ermilov \& Anichkin, 2014 [Ermilov and Anichkin 2014b]

G. (Galumna) paramastigophora Ermilov, 2015 [Ermilov 2015a]

G. (Galumna) pseudokhoii Ermilov \& Anichkin, 2011 [Ermilov and Anichkin 2011h]

G. (Galumna) pseudotriquetra Ermilov, 2015 [Ermilov 2015a]

G. (Galumna) triquetra Aoki, 1965 [Vu 1990] 
G. (Galumna) triops Balogh, 1960 [Vu et al. 2015]

G. (Cosmogalumna) dongnaiensis Ermilov \& Anichkin, 2013 [Ermilov and Anichkin 2013f]

G. (Cosmogalumna) praeoccupata Subías, 2004 [Ermilov and Anichkin 2014d]

G. (Cosmogalumna) tenensis Ermilov, Vu \& Nguyen, 2011 [Ermilov et al. 2011b]

G. (Neogalumna) longilineata Ermilov \& Anichkin, 2014 [Ermilov and Anichkin 2014d]

G. (Neogalumna) seniczaki Ermilov \& Anichkin, 2010 [Ermilov and Anichkin 2010]

G. (Neogalumna) tolstikovi Ermilov \& Anichkin, 2014 [Ermilov and Anichkin 2014d]

Leptogalumna (Leptogalumna) ciliata Balogh, 1960 [Ermilov and Anichkin 2011h]

Notogalumna foveolata Balakrishnan, 1989 [Ermilov and Anichkin 2013g]

N. lagunaensis Ermilov \& Corpuz-Raros, 2015 [Ermilov and Corpuz-Raros 2015]

Pergalumna (Pergalumna) altera (Oudemans, 1915) [Vu et al. 1985]

P. (Pergalumna) cattienica Ermilov \& Anichkin, 2011 [Ermilov and Anichkin 2011f]

P. (Pergalumna) granulata Balogh \& Mahunka, 1967 [Balogh and Mahunka 1967]

P. (Pergalumna) hauseri Mahunka, 1995 [Ermilov and Anichkin 2013d]

P. (Pergalumna) indistincta Ermilov \& Anichkin, 2011 [Ermilov and Anichkin 2011h]

P. (Pergalumna) kotschyi Mahunka, 1989 [Mahunka 1989]

P. (Pergalumna) longisetosa Balogh, 1960 [Vu et al. 2014]

P. (Pergalumna) magnipora capillaris Aoki, 1961 [Vu 1993]

P. (Pergalumna) margaritata Mahunka, 1989 [Mahunka 1989]

P. (Pergalumna) mauritii Mahunka, 1978 [Vu et al. 2014]

P. (Pergalumna) montana Hammer, 1961 [Vu et al. 2015]

P. (Pergalumna) nuda Balogh, 1960 [Vu et al. 2014]

P. (Pergalumna) paraelongata Ermilov \& Anichkin, 2012 [Ermilov et al. 2012c]

P. (Pergalumna) pseudosejugalis Ermilov \& Anichkin, 2012 [Ermilov and Anichkin 2012d]

P. (Pergalumna) punctulata Balogh \& Mahunka, 1967 [Balogh and Mahunka 1967]

P. (Pergalumna) taprobanica Balogh, 1988 [Vu et al. 2014]

P. (Pergalumna) yurtaevi Ermilov \& Anichkin, 2011 [Ermilov and Anichkin 2011f]

Trichogalumna nipponica (Aoki, 1966) [Ermilov et al. 2012c]

T. subnuda Balogh \& Mahunka, 1967 [Balogh and Mahunka 1967]

T. vietnamica Mahunka, 1987 [Mahunka 1987]

\section{Galumnellidae}

Galumnella (Galumnella) cellularis (Balogh \& Mahunka, 1967) [Balogh and Mahunka 1967]

G. (Galumnella) geographica Mahunka, 1995 [Vu et al. 2014] 
G. (Galumnella) microporosa Ermilov \& Anichkin, 2011 [Ermilov and Anichkin 2011f]

G. (Galumnella) tiunovi Ermilov \& Anichkin, 2013 [Ermilov and Anichkin 2013d]

G. (Galumnella) paulinai Balogh, 1961 [Vu et al. 2014]

G. (Bagalumnella) scavasorum (Mahunka, 1994) [Vu et al. 2014]

Porogalumnella pulchella Aoki \& Hu, 1993 [Ermilov and Anichkin 2014d]

\section{Conclusion}

The list of oribatid mites of Vietnam includes now 535 species/subspecies, 222 genera and 81 families. Of these, 194 species/subspecies were described as new for science from Vietnam; 94 species have been described by Ermilov and co-authors, 30 species by Balogh and Mahunka, 28 species by Mahunka, 21 species by Niedbała, 6 species by Jeleva and $\mathrm{Vu}, 4$ species by Fernandez and co-authors, 3 species by Krivolutsky, 2 species by Golosova, 2 species by Starý, 2 species by Vu and co-authors, 1 species by Balogh, 1 species by Rajski and Szudrowicz.

\section{Acknowledgements}

The author cordially thanks Prof. Dr. Badamdorj Bayartogtokh (National University of Mongolia, Ulaanbaatar, Mongolia) and Prof. Dr. Gerd Weigmann (Free University of Berlin, Institute of Zoology, Berlin, Germany) for valuable comments, Prof. Dr. Roy A. Norton (State University of New York, Syracuse, U.S.A.), Kerstin Franke (Senckenberg Museum für Naturkunde Görlitz, Germany) and Duy Dinh (Joint Russian-Vietnamese Tropical Research and Technological Center, Hanoi-Ho Chi Minh, Vietnam) for help with collecting literature. The study on the oribatid mite fauna was supported by the Russian Foundation for Basic Research (project 14-04-31183 mol_a to Dr. S.G. Ermilov) and the Ministry of Education and Science of the Russian Federation (project No. 6.1933.2014/K, code 1933 to Dr. A.V. Tolstikov). The study on moss mites was supported by the Russian Science Foundation (project 14-14-01134 to Dr. A.A. Prokin). The taxonomic study on Galumnoidea was supported by the Russian Foundation for Basic Research (project: 15-04-02706 A to Dr. S.G. Ermilov).

\section{References}

Anichkin AE, Ermilov SG, Tolstikov AV (2014) The catching belt. Patent, useful model, № 138455 (demand № 2013135917, 30 July 2013, registered 18.02.2014), 2 pp.

Balogh J (1983) A partial revision of the Oppiidae Grandjean, 1954 (Acari: Oribatei). Acta Zool Acad Sci Hung 29(1-3): 1-79. 
Balogh J, Mahunka S (1967) New oribatids (Acari) from Vietnam. Acta Zool Acad Sci Hung 13(1-2): 39-74.

Ermilov SG (2011) A new genus and species of Amerobelbidae (Acari, Oribatida) from Vietnam. Acarologia 51(3): 275-282. doi: 10.1051/acarologia/20112012

Ermilov SG (2013) Additional description of Scheloribates (Bischeloribates) mahunkai Subías, 2010 (Acari: Oribatida: Scheloribatidae). Opusc Zool Budapest 44 (1): 9-14.

Ermilov SG (2015a) New and little known species of oribatid mites of the subgenus Galumna (Galumna) (Acari, Oribatida, Galumnidae) from Vietnam. Zootaxa 3946 (4): 553-566.

Ermilov SG (2015b) Two new species of oribatid mites (Acari, Oribatida) from southern Vietnam. Acarina 23(1): 55-62.

Ermilov SG, Anichkin AE (2010) Three new species of Galumnidae (Acari: Oribatida) from Cat Tien National Park, southern Vietnam. Zootaxa 2681: 20-34.

Ermilov SG, Anichkin AE (2011a) A new diagnosis of Schalleriella (Acari: Oribatida: Microzetidae) and a new species from Vietnam. Syst Appl Acarol 16(2): 169-175.

Ermilov SG, Anichkin AE (2011b) A new species of Arthrodamaeus from Vietnam (Acari: Oribatida: Gymnodamaeidae). Genus 22(1): 151-159.

Ermilov SG, Anichkin AE (2011c) A new species of Protoribates (Acari: Oribatida: Haplozetidae) from southern Vietnam. Int J Acarol 37 (Suppl 1): 244-251.

Ermilov SG, Anichkin AE (2011d) Eremulus spinosus, a new species of oribatid mite from Vietnam (Acari: Oribatida: Eremulidae). Genus 22(4): 645-651.

Ermilov SG, Anichkin AE (2011e) Four new species of oribatid mites (Acari: Oribatida) from Vietnam. Zoosyst Ros 20 (2): 200-215.

Ermilov SG, Anichkin AE (2011f) New oribatid mites of the genera Pergalumna and Galumnella (Acari, Oribatida, Galumnoidea) from Vietnam. Acarina 19(2): 242-251.

Ermilov SG, Anichkin AE (2011g) New oribatid mites of the genera Pulchroppia and Lineoppia (Acari: Oribatida: Oppiidae) from Cat Tien National Park in southern Vietnam. Acarologia 51(1): 31-42.

Ermilov SG, Anichkin AE (2011h) The galumnoid fauna (Acari: Oribatida) of Cat Tien National Park (southern Vietnam) with description of two new species. Int J Acarol 37 (Suppl 1): 85-94.

Ermilov SG, Anichkin AE (2011i) The oribatid mite families Nanhermanniidae and Lohmanniidae of Cat Tien National Park (Vietnam). Acarina 19 (2): 231-241.

Ermilov SG, Anichkin AE (2011j) Three new species of Haplozetidae (Acari: Oribatida) from Vietnam. Acarologia 51(1): 43-59.

Ermilov SG, Anichkin AE (2011k) Two new species of Oripodoidea (Acari: Oribatida) from Vietnam. Acarologia 51(2): 143-154.

Ermilov SG, Anichkin AE (2012a) A new species of Oribatella (Acari: Oribatida: Oribatellidae) from Vietnam, including a key to species of the genus from the Oriental region. Int J Acarol 38(4): 301-307.

Ermilov SG, Anichkin AE (2012b) Oribatid mites of the genera Epilohmannia, Furcoppia and Unguizetes (Acari: Oribatida: Epilohmanniidae; Astegistidae; Mochlozetidae) from Vietnam. Syst Appl Acarol 17(1): 91-105. 
Ermilov SG, Anichkin AE (2012c) Two new oribatid mite species with auriculate pteromorphs from Southern Vietnam (Acari: Oribatida: Parakalummidae, Galumnidae). Opusc Zool Budapest 43(2): 161-167.

Ermilov SG, Anichkin AE (2012d) Two new species of oribatid mites (Acari: Oribatida) from Bu Gia Map National Park (Vietnam). Zoosyst Ros 21(1): 18-27.

Ermilov SG, Anichkin AE (2013a) A new species of Plakoribates (Acari: Oribatida: Achipteriidae) from Vietnam. Syst Appl Acarol 18 (2): 137-144.

Ermilov SG, Anichkin AE (2013b) A new species of Ramuselloppia (Acari: Oribatida: Oppiidae) from Vietnam. Pers J Acarol 2(1): 25-33.

Ermilov SG, Anichkin AE (2013c) Checklist of oribatid mites (Acari: Oribatida) from two forest plantations of Southern Vietnam, including new records and description of a new species of the genus Suctobelbata (Suctobelbidae). Syst Appl Acarol 18(3): 225-232.

Ermilov SG, Anichkin AE (2013d) Collection of oribatid mites (Acari: Oribatida) from Dong Nai Biosphere Reserve of Southern Vietnam, with description of three new species. Ann Zool 63(2): 177-193.

Ermilov SG, Anichkin AE (2013e) Oribatid mites (Acari: Oribatida) from acacia and pine plantations in southern Vietnam, with description of a new species of the subgenus $G a-$ lumna (Cosmogalumna). Syst Appl Acarol 18(1): 80-88.

Ermilov SG, Anichkin AE (2013f) Oribatid mites (Acari, Oribatida) from dipterocarp and polydominant forests of the Dong Nai Culture and Nature Reserve (Southern Vietnam), with description of a new species of Lyroppia (Oppiidae). Acarologia 53(1): 101-109.

Ermilov SG, Anichkin AE (2013g) Oribatid mites (Acari: Oribatida) of fungi from Dong Nai Biosphere Reserve, Southern Vietnam. Pers J Acarol 2(2): 195-208.

Ermilov SG, Anichkin AE (2014a) A new species of Dimidiogalumna (Acari: Oribatida: Galumnidae) from Vietnam, including a key to all species of the genus. Syst Appl Acarol 19(1): 67-72.

Ermilov SG, Anichkin AE (2014b) A new species of Galumna (Galumna) (Acari, Oribatida, Galumnidae) from Vietnam. Ecol Mont 1(1): 9-14.

Ermilov SG, Anichkin AE (2014c) A new species of Scheloribates (Scheloribates) from Vietnam, with notes on taxonomic status of some taxa in Scheloribatidae (Acari, Oribatida). Int J Acarol 40(1): 109-116.

Ermilov SG, Anichkin AE (2014d) Taxonomic study of oribatid mites (Acari, Oribatida) of Bi Dup - Nui Ba National Park (southern Vietnam). Zootaxa 3834(1): 1-86.

Ermilov SG, Anichkin AE (2014e) Two new species of oribatid mites of the family Galumnidae (Acari, Oribatida) from Vietnam. ZooKeys 382: 53-66.

Ermilov SG, Anichkin AE (2014f) Two new species of Pedrocortesella, with the checklist of oribatid mites from riverine substrata in southern Vietnam (Acari, Oribatida, Pedrocortesellidae). Spixiana 37(2): 207-218.

Ermilov SG, Anichkin AE (2014g) Vietnamese oribatid mites of the genus Dolicheremaeus (Acari, Oribatida, Tetracondylidae), with description of two new species. Syst Appl Acarol 19(2): 205-215. 
Ermilov SG, Anichkin AE (2015a) A new oribatid mite species (Acari, Oribatida) from a mangrove forest of southern Vietnam. Zool Zh 94(6): 651-660. [Eng vers: Ent Rev 2015. 95(5): 672-680.] doi: 10.1134/S0013873815050115

Ermilov SG, Anichkin AE (2015b) A new species of oribatid mites of the genus Scapheremaeus (Acari, Oribatida, Cymbaeremaeidae) from Vietnam. Zool Zh 94(4): 488-493.

Ermilov SG, Anichkin AE, Wu D (2012a) Oribatid mites from Bu Gia Map National Park (Southern Vietnam), with description of a new species of Dolicheremaeus (Tetracondylidae) (Acari: Oribatida). Genus 23(4): 591-601.

Ermilov SG, Anichkin AE, Wu D (2012b) Two new species of the genus Papillacarus (Acari: Oribatida: Lohmanniidae) from caves of Southern Vietnam. Zootaxa 3593: 75-88.

Ermilov SG, Anichkin AE, Palko IV (2013a) Oribatid mites (Acari) from nests of some birds in South Vietnam. Zool Zh 92(7): 802-807. [Eng vers: Ent Rev 2013. 93 (6): 799-804.] Ermilov SG, Anichkin AE, Wu D (2013b) A new oribatid mite species of the genus Sphaerozetes (Acari, Ceratozetidae) from Vietnam. Zool Zh 92(8): 986-990. [Eng vers: Ent Rev 2014. 94 (1): 131-135.] doi: 10.7868/S0044513413080060

Ermilov SG, Aoki J, Anichkin AE (2013c) Description of Chistyakovella insolita gen. nov., sp. nov., and redescription of the type species of Diplobodes, D. kanekoi Aoki, 1958 (Acari: Oribatida: Carabodidae). Zootaxa 3608(3): 178-190. doi: 10.11646/zootaxa.3608.3.2

Ermilov SG, Anichkin AE, Tolstikov AV (2014a) A new species of oribatid mites of the genus Malaconothrus (Acari, Oribatida, Malaconothridae) from Vietnam. Acarina 22(1): 20-23. doi: 10.7868/S0044513413070040

Ermilov SG, Anichkin AE, Tolstikov AV (2014b) The oribatid mite genus Papillocepheus (Acari, Oribatida, Tetracondylidae), with description of a new species from southern Vietnam. ZooKeys 381: 1-10. doi: 10.3897/zookeys.381.6832

Ermilov SG, Anichkin AE, Tolstikov AV (2014c) Umashtanchaeviella plethotricha, a new genus and species of the family Tetracondylidae (Acari, Oribatida). ZooKeys 408: 51-59. doi: 10.3897/zookeys.408.7605

Ermilov SG, Shtanchaeva UYa, Subías LS (2014d) New species of oribatid mites (Acari: Oribatida) of the genera Suctobelbella (Suctobelbidae) and Neoribates (Parakalummidae) from Vietnam. Biologia 69(11): 1593-1600. doi: 10.2478/s11756-014-0457-6

Ermilov SG, Shtanchaeva UYa, Subías LS, Anichkin AE (2014e) A new subgenus and three new species of oribatid mites of the genus Yoshiobodes (Acari, Oribatida, Carabodidae) from Vietnam. Zootaxa 3795(4): 401-420. doi: 10.11646/zootaxa.3795.4.1

Ermilov SG, Bayartogtokh B (2015) The oribatid mite fauna (Acari, Oribatida) of the Bu Gia Map National Park (southern Vietnam), with description of two new species. Int J Acarol 41(3): 220-231. doi: 10.1080/01647954.2015.1026393

Ermilov SG, Corpuz-Raros LA (2015) A new species of Notogalumna (Acari, Oribatida, Galumnidae) from the Oriental region a key to known species. Acarologia 55 (3): 267-276. doi: 10.1051/acarologia/20142168

Ermilov SG, Niedbała W (2013) Contribution to the knowledge of the oribatid mite fauna of Bolivia, Zambia, Cambodia and Vietnam, with descriptions of two new species (Acari: Oribatida). Spixiana 36(1): 9-19. 
Ermilov SG, Niedbała W, Anichkin AE (2012c) Oribatid mites of Dong Nai Biosphere Reserve (=Cat Tien National Park) of Southern Vietnam, with description of a new species of Pergalumna (Acari, Oribatida, Galumnidae). Acarina 20(1): 20-28.

Ermilov SG, Ryabinin NA, Anichkin AE (2012d) The morphology of the juvenile stages of two oribatid species of the family Hermanniidae (Oribatida). Zool Zh 91(6): 657-668. [Eng vers: Ent Rev 2012. 92 (7): 815-826.]

Ermilov SG, Shimano S, Vu QM (2011a) Redescription of Papillacarus hirsutus with remarks on taxonomic status of Papillacarus arboriseta (Acari: Oribatida: Lohmanniidae). Acarologia 51(2): 155-163. doi: 10.1051/acarologia/20112003

Ermilov SG, Shtanchaeva UYa, Subías LS, Anichkin AE (2012e) Morphology of juvenile instars of Meristacarus sundensis Hammer, 1979 and Cryptacarus promecus Grandjean, 1950 (Acari, Oribatida, Lohmanniidae). Syst Appl Acarol 17(3): 281-300. doi: 10.11158/ saa.17.3.8

Ermilov SG, Shtanchaeva UYa, Subías LS, Anichkin AE (2014f) The family Ctenobelbidae (Acari, Oribatida), with description of a new species and discussion on systematic placement and taxonomic status of the genus Berndamerus Mahunka, 1977. ZooKeys 395: 1-10. doi: 10.3897/zookeys.395.7224

Ermilov SG, Shtanchaeva UYa, Subías LS, Anichkin AE (2012f) The oribatid mite genus Hammerella, with description of a new subgenus and species from Vietnam (Acari: Oribatida: Granuloppiidae). Acarina 20(2): 159-166.

Ermilov SG, Vu QM (2012) Two new species of oribatid mites (Acari: Oribatida) from Phong Nha-Ke Bang National Park of central Vietnam. Int J Acarol 38(2): 160-167. doi: 10.1080/01647954.2011.603497

Ermilov SG, Vu QM, Nguyen HT (2011b) Galumna (Cosmogalumna) tenensis, a new species of oribatid mite from northwestern Vietnam (Acari: Oribatida: Galumnidae). Int J Acarol 37 (Suppl 1): 53-60. doi: 10.1080/01647954.2010.537697

Ermilov SG, Vu QM, Trinh TT, Dao DT (2011c) Perxylobates thanhoaensis, a new species of oribatid mite from Vietnam (Acari: Oribatida: Haplozetidae). Int J Acarol 37(2): 161-166. doi: 10.1080/01647954.2010.496374

Fernandez N, Theron P, Rolland C, Castillo ER (2015a) Oribatid mites from deep soils of Hòn Chông limestone hills, Vietnam: the family Lohmanniidae (Acari: Oribatida), with the descriptions of Bedoslohmannia anneae n. gen., n. sp., and Paulianacarus vietnamese n. sp. Zoosystema 36(4): 771-187. doi: 10.5252/z2014n4a5

Fernandez N, Theron P, Rolland C, Leiva S (2015b) Oribatid mites (Acari: Oribatida) from deep soils of Hòn Chông limestone hills, Kien Giang Province, Vietnam. II. Descriptions of two new species, Papillacarus whitteni sp. nov. (family Lohmanniidae) and Basilobelba maidililae s. n. (family Basilobelbidae). Int J Acarol 41(2): 132-146. doi: 10.1080/01647954.2015.1014414

Golosova LD (1983) Some notes about oribatid mites of Vietnam. In: Golosova LD (Ed.) Ecology and fauna of animals. Tyumen State Univ Press, Tyumen, 41-51.

Golosova LD (1984) Two new species of oribatid mites from Vietnam. Zool Zh 63(4): 620 621. 
Jeleva M, Vu QM (1987) New Oribatids (Oribatei, Acari) from the Northern part of Vietnam. Acta Zool Bulg 33: 10-18.

Krivolutsky DA (1998) Oribatid mites of the family Nothridae from mountains of North

Vietnam. In: Chan ST, Kalyakin MV, Korzun LP, Kuznetsov AN, Phang L, Roumak VS,

Rozhnov VV (Eds) Mat Zool Bot Stud Fan Si Pan summit area (North Vietnam). Rus Acad Sci, Moscow, 255-261.

Mahunka S (1987) A survey of the Oribatid (Acari) fauna of Vietnam, I. Ann Hist-Nat Mus Nat Hung 79: 259-279.

Mahunka S (1988) A survey of the Oribatid Fauna (Acari) of Vietnam, II. Acta Zool Hung 34(2-3): 215-246.

Mahunka S (1989) A survey of the Oribatid fauna (Acari) of Vietnam, III. Folia Ent Hung 50: 47-59.

Nguyen HT, Vu QM (2012) Species composition of Oribatida in Phong Nha-Ke Bang National Park, Quang Binh Province. J Sci, Nat Sci Trch, Viet Nat Univ, Hanoi 28(2): 125-134. Nguyen HT, Lai TH, Vu QM, Nguyen HT (2013) Distribution characteristics of oribatid mite community (Acari: Oribatida) according to seasons and habitats in Cat Ba National Park, Hai Phong City. Proc 5th Nat Sci Conf Ecol Biol Res 5: 1673-1678.

Niedbała W (1989) Phthiracaroidea (Acari, Oribatida) nouveaux de la région orientale. Ann Zool 43(1): 1-13.

Niedbała W (2000) The ptyctimous mites of the Oriental and Australian regions and their centers of its origin (Acari: Oribatida). Genus (Suppl): 1-493.

Niedbała W (2004) Supplement to the knowledge of ptyctimous mites of the Oriental region (Acari, Oribatida). Genus 15(3): 391-423.

Niedbała W, Ermilov SG (2013) Ptyctimous mites (Acari, Oribatida) from Southern Vietnam with descriptions of three new species. Zootaxa 3608(6): 521-530. doi: 10.11646/zootaxa.3608.6.5

Niedbała W, Ermilov SG (2014) Ptyctimous mites (Acari, Oribatida) from the Joint RussianVietnamese Biological Expedition (October 2013-April 2014). Zootaxa 3884(2): 156168. doi: 10.11646/zootaxa.3884.2.4

Norton RA, Behan-Pelletier VM (2009) Chapter 15. Oribatida. In: Krantz GW, Walter DE (Eds) A Manual of Acarology. Texas Tech Univ Press, Lubbock, 430-564.

Pavlichenko PG (1991) A new finding of ceratozetoid mite (Oribatei, Ceratozetoidea) in Vietnam. Vest Zool 1: 87.

Rajski A, Szudrowicz R (1974) Oribatei (Acari) from Northern Vietnam. I. Dolicheremaeus bartkei sp. n. Acta Zool Cracov 19(11): 217-232.

Schatz H, Behan-Pelletier VM, OConnor BM, Norton RA (2011) Suborder Oribatida van der Hammen, 1968. In: Zhang Z-Q (Ed.) Animal biodiversity: An outline of higher-level classification and survey of taxonomic richness. Zootaxa 3148: 141-148.

Starý J (1993) New species of the genus Euphthiracarus (Acari: Oribatida) from Vietnam. Acta Soc Zool Bohem 56(4): 295-305.

Subías LS (2004) Listado sistemático, sinonímico y biogeográfico de los ácaros oribátidos (Acariformes: Oribatida) del mundo (excepto fósiles). Graellsia 60 (número extraordinario): 3-305. Online version accessed in March 2015, 587 pp. 
Vu QM (1990) The investigations on microarthropods of the soil zoocoenoses in Vietnam. J Biol 12(1): 3-10.

Vu QM (1993) A contribution to the studies of oribatid mites (Acari: Oribatei) in the hillymountains north-west zone of Vietnam. J Biol 15(4): 66-68.

Vu QM (1994) Data on the oribatid community structures (Acari: Oribatei) of the Cat Ba island and related seaside areas, North Vietnam. In: Pham TTC (Ed.) Sci Bul Univ, Biol seminar-Agr: Min Ed Train, Dept Tech, Hanoi, 14-19.

Vu QM (2007) Fauna of Vietnam, 21, Oribatida. Sci. and Tech Publ House, Hanoi, 355.

Vu QM (2013) The oribatid fauna (Acari: Oribatida) of Vietnam - Systematics, zoogeography and zonation, formation and role in the soil ecosystem. Dissertation abstract. Sofia, 56 pp.

Vu QM, Ermilov SG, Dao TD (2010) Two new species of Oribatid Mites (Acari: Oribatida) from Vietnam. J Biol TCSH 32(3): 12-18.

Vu QM, Jeleva M, Tsonev I (1985) Faunal-Ecological studies on oribatid mites (Oribatei, Acari) in agroecosystems in the northern Vietnam. Proc Conf Ecol Environ Prot, Sofia, Bulg, 93-102.

Vu QM, Jeleva M, Tsonev I (1987) Oribatid Mites (Oribatei, Acarina) of the plain of the Red river in Vietnam. In: Striganova BR (Ed.) Soil Fauna and Soil Fertility. Nauka, Moscow, 601-604.

Vu QM, Luu TN, Nguyen HT (2008) Soil microarthropod community structures (Microarthropoda: Oribatida, Collembola) in relation to biotope's type in the Red river plain, Vietnam. J Sci Tech Agr Vietnam 5(6): 81-86.

Vu QM, Ngo NH, Nguyen HT (2011) Oribatid mites (Acari: Oribatida) in the National Parks of Ben En (Thanh Hoa) and Phong Nha-Ke Bang (Quang Binh) and related areas. Proc $4^{\text {th }}$ Nat Sci Conf Ecol Biol Resources, 214-219.

$\mathrm{Vu}$ QM, Ngo NH, Nguyen HT (2012) Oribatid mites (Acari: Oribatida) community in the soil ecosystem of Nui che, Tien Du District, Bac Ninh Province, the upland area of northern Vietnam. HNUE J Sci 57(3): 110-118.

$\mathrm{Vu}$ QM, Nguyen TT (2000) Microarthropod community structures in Tam Dao National Park, Vietnam. J Biosci 25(4): 379-386. doi: 10.1007/BF02703791

Vu QM, Nguyen XL (2005) Distributional and geographical remarks on oribatid fauna (Acari, Oribatida) in Vietnam. Proc 5th Viet Nat Conf Ent, Hanoi, ESOV, 137-143.

Vu MQ, Nguyen TH, Lai TH (2014) Species diversity of Oribatida community (Acari, Oribatida) in the soil ecosystem of the Cat Ba National Park, Northern Vietnam. Proc 8th Viet Conf Ent, 916-928.

Vu QM, Nguyen HT, Tran TT, Do TH (2015) Soil oribatid mite (Acari: Oribatida) community structure as a bioindicator of seasonal climate conditions in Cuc Phuong National Park, Ninh Binh Province. J Sci HNUE Nat Sci 60(4): 80-86.

Vu QM, Thi HV (1995) The list of oribatid mites from Vietnam. J Biol 17(3): 49-55.

Weigmann G (2006) Hornmilben (Oribatida). Die Tierwelt Deutschlands. Teil 76. Keltern, Goecke and Evers, 520 pp. 